Revue internationale P.M.E.

Économie et gestion de la petite et moyenne entreprise

\title{
Les entreprises à forte croissance et leur contribution à l'emploi : le cas de la Suède 1987-1996
}

Per Davidsson et Frédéric Delmar

Volume 14, numéro 3-4, 2001

URI : https://id.erudit.org/iderudit/1008701ar

DOI : https://doi.org/10.7202/1008701ar

Aller au sommaire du numéro

Éditeur(s)

Presses de l’Université du Québec

ISSN

0776-5436 (imprimé)

1918-9699 (numérique)

Découvrir la revue

Citer cet article

Davidsson, P. \& Delmar, F. (2001). Les entreprises à forte croissance et leur contribution à l'emploi : le cas de la Suède 1987-1996. Revue internationale P.M.E., 14(3-4), 163-187. https://doi.org/10.7202/1008701ar
Résumé de l'article

Cette étude explore la manière dont les entreprises à forte croissance en Suède contribuent à la création d'emplois. Avec un échantillon composé de toutes les entreprises en Suède ayant plus de 20 employés et en activité en 1996 ( $n$ = 11 748), nous avons analysé leurs développements annuels au cours des dix dernières années (soit de 1987 à 1996). Nous avons défini un échantillon d'entreprises à forte croissance comprenant les $10 \%$ qui ont le plus contribué en moyenne annuelle absolue à la croissance d'emplois $(n=1153)$. Les résultats indiquent que les entreprises jeunes et de taille moyenne, souvent dans des industries jeunes et en croissance, créent la majeure partie des nouveaux emplois. Néanmoins, la contribution totale à la création d'emplois par les entreprises en forte croissance est inférieure à celle des entreprises récemment établies. Des implications pour la recherche et les pratiques sont offertes.
Ce document est protégé par la loi sur le droit d'auteur. L'utilisation des services d’Érudit (y compris la reproduction) est assujettie à sa politique d'utilisation que vous pouvez consulter en ligne.

https://apropos.erudit.org/fr/usagers/politique-dutilisation/ 


\title{
Les entreprises à forte croissance et leur contribution à l'emploi: le cas de la Suède 1987-1996
}

\author{
Per DAVIDSSON \\ École internationale des affaires de Jönköping \\ Frédéric DELMAR \\ Centre d'entrepreneuriat de l'École de sciences économiques de Stockholm
}

\section{MOTS CLÉS}

\section{Croissance d'entreprises - Croissance interne - Acquisition Cycle conjucturel - Étude longitudinale}

\section{RÉSUMÉ}

Cette étude explore la manière dont les entreprises à forte croissance en Suède contribuent à la création d'emplois. Avec un échantillon composé de toutes les entreprises en Suède ayant plus de 20 employés et en activité en 1996 ( $n=11$ 748), nous avons analysé leurs développements annuels au cours des dix dernières années (soit de 1987 à 1996). Nous avons défini un échantillon d'entreprises à forte croissance comprenant les $10 \%$ qui ont le plus contribué en moyenne annuelle

\section{LES AUTEURS}

PER DAVIDSSON est professeur d'administration des affaires avec spécialisation en entrepreneuriat à la Jönköping International Business School. Depuis qu'il a rédigé sa thèse de doctorat en 1989 intitulée Continued Entrepreneurship and Small Firm Growth, la croissance des entreprises est un thème central dans sa recherche. Adresse : P.O. Box 1026, SE-551 11 Jönköping, Suède. Téléphone : 4636 15 6430. Courriel : <per.davidsson@jibs.hj.se>. FRÉDÉRIC DELMAR est professeur associé à la Stockholm School of Economics avec spécialisation en entrepreneuriat. Depuis qu'il a rédigé sa thèse de doctorat en 1996 qui portait sur Entrepreneurial Behavior and Business Performance, il s'est intéressé à la croissance des entreprises et au processus de création des entreprises. Adresse: P.O. Box 6501, SE-113 83 Stockholm, Suède. Téléphone : 468736 9356, télécopieur: 46831 2785. Courriel : <frederic.delmar@hhs.se>. 
absolue à la croissance d'emplois ( $\mathrm{n}=1153$ ). Les résultats indiquent que les entreprises jeunes et de taille moyenne, souvent dans des industries jeunes et en croissance, créent la majeure partie des nouveaux emplois. Néanmoins, la contribution totale à la création d'emplois par les entreprises en forte croissance est inférieure à celle des entreprises récemment établies. Des implications pour la recherche et les pratiques sont offertes.

\begin{abstract}
This study explores how high-growth firms in Sweden contribute to job creation. Using the population of all firms in Sweden with more than 20 employees in existence in 1996 ( $\mathrm{n}=11,748)$, we analysed their annual development for the previous ten years (1987 to 1996). We defined as a sub-sample of high growth firms those $10 \%$ of all firms that contributed most in annual average absolute employment growth ( $\mathrm{n}=1153$ ). The results indicate that young and medium sized firms, often in young and growing industries, create new jobs to a great extent. However, the total contribution to job creation by high growth firms is less than that of newly established firms. Implications for research and practice are offered.

\section{RESUMEN}

Este estudio explora la contribución de las empresas de alto crecimiento a la creación de empleo en Suecia. Usando la población completa de las empresas Suecas en existencia en 1996 con más de 20 empleados, analizamos su desarollo annual, por cada año, durante los últimos 10 años (1987-1996). De ésta población de todas las empresas en Suecia, definimos cómo sub-selección de las empresas de alta crecimiento al $10 \%$ que contribueron más al medio crecimiento de empleo absoluto sobre una base annual $(\mathrm{n}=1153)$. Los resultados indican que las empresas jovenes y medianas, a menudo provenidas de industrias jovenes y en crecimiento, crean la mayor parte de los nuevos trabajos. No obstante, la contribución a la creación de empleo total por las empresas de alto crecimiento, es inferior que aquella de las empresas recientimento establecidas. Implicaciones para la investigación y la practica son ofrecidas.
\end{abstract}

\title{
ZUSAMMENFASSUNG
}

Diese Untersuchung erklärt, wie die stark wachsenden Unternehmen in Schweden zur Arbeitsplatzgründung beitragen. Bei allen schwedischen Unternehmen mit mehr als 20 Mitarbeitern des Jahres 1996 ( $\mathrm{n}=11748$ ) haben wir die jährlichen Entwicklungen für die vergangenen 10 Jahre analysiert (von 1987 bis 1996). Wir haben eine Stichprobe von $10 \%$ stark wachsenden Unternehmen definiert, die ein Beschäftigtenwachstum über dem absoluten jährlichen Durchschnitt aufweisen ( $\mathrm{n}=1153$ ). Die Ergebnisse zeigen auf, dass die jungen Unternehmen von mittlerer Grösse, oft in jungen Industrien und im Wachstum, am meisten zur Gründung von Arbeitsplätzen beitragen. Nichtsdestoweniger ist der gesamte Beitrag an der Gründung von Arbeitsplätzen durch die stark wachsenden Unternehmen geringer als derjenige, der vor kurzem gegründeten Unternehmen. 


\section{Introduction}

La dynamique de création d'emplois a, au cours des dernières années, attiré l'attention des chercheurs ainsi que des politiciens. Un bon nombre d'études réalisées dans différents pays indiquent que les PME jouent un rôle important ou croissant comme créateur d'emplois (Birch, 1979; Baldwin et Picot, 1995 ; Davidsson, 1995 ; Davidsson, Lindmark et Olofsson, 1993, 1994, 1995, 1997 a et b; Fumagelli et Mussati, 1993 ; Kirchhoff et Phillips, 1988 ; Picot et Dupuy, 1998 ; Spilling, 1995 ; pour d'autres études réalisées dans un grand nombre de pays voir aussi Aiginger et Tichy, 1991 ; ENSR, 1994 ; Loveman et Sengenberger, 1991 ; OCDE, 1987 ; Storey et Johnson, 1987).

Alors qu'une majorité de chercheurs conviennent de l'importance des PME, on continue de discuter leur rôle en tant que créateurs d'emplois : est-ce surtout le résultat du grand nombre de création d'entreprises et de leur croissance par la suite ou si une minorité d'entreprises à très forte croissance créent la majeure partie des emplois? Cela est connu comme le débat des souris contre les gazelles. Storey (1994), Storey et Johnson (1987) et Birch et Medoff (1994) défendent fortement la position que ce petit groupe d'entreprises à forte croissance représente les « vrais » créateurs d'emplois aux États-Unis et en Grande-Bretagne. Les études menées par Davidsson et ses collaborteurs en Suède confirment plutôt l'hypothèse des souris.

Ces différentes perspectives s'expliquent en partie par des différences entre pays, mais on doit aussi comprendre que ces disparités dans les résultats sont aussi dues en grande partie à des différences de méthode. Comme le soulignent Davis, Haltiwanger et Schuh (1993, 1996), la création nette d'emplois dans une économie peut être attribuée à plusieurs sous-groupes d'entreprises. Aussi, certaines études analysent le développement historique d'un groupe d'entreprises sans prendre en considération les entreprises créées ou fermées pendant la période d'observation. Dans ce cas, si quelques entreprises ferment pendant la période à l'étude et que les autres suivent des rythmes de croissance variés, avec le temps, une petite fraction du groupe original comptera pour une partie toujours croissante des emplois totaux du groupe. D'un autre côté, si les études tiennent compte de toutes les créations d'entreprises, des fermetures, des expansions et des contractions, mais n'agrègent pas la contribution individuelle des entreprises pendant la période d'observation, le résultat typique sera que la somme de plusieurs petites contributions aura des effets importants.

La contribution des PME en forte croissance à l'emploi dépend aussi largement de la façon dont cette catégorie est définie. Une définition basée sur la croissance du chiffre d'affaires, cetirus paribus, aura l'effet d'y attribuer une moindre part de la croissance d'emploi, par comparaison avec une définition basée sur la croissance en emploi. Une définition basée sur la croissance relative favorisera les plus petites entreprises mais attribuera une part plus petite de la croissance totale

Revue internationale P.M.E., vol. 14, $\mathrm{n}^{\text {os }}$ 3-4, 2001 
de l'emploi à cette catégorie qu'une définition basée sur la croissance absolue de l'emploi au niveau de l'entreprise. Il est donc important de réaliser que les résultats sont en partie reliés à la méthodologie et aux définitions employées dans les études.

Toutefois, la majorité des chercheurs et des politiciens sont probablement en accord avec les propositions suivantes :

- le petit groupe d'entreprises à forte croissance (EFC) a un impact très important sur la création d'emplois ou, du moins, l'aurait si le développement d'un tel groupe pouvait être stimulé;

- l'intérêt pour les (petites et moyennes) entreprises à forte croissance a augmenté dramatiquement ces dernières années, en témoingne le nombre d'études récentes sur leurs caractéristiques et occurrence (Blixt, 1997; Gundry et Welsch, 2001 ; Johnson, Baldwin et Hinchley, 1997 ; Sexton et Seale, 1997; Vyakarnam, Jacobs et Handelberg, 1997);

- nos connaissances actuelles sur la contribution économique et les pratiques de gestion des PME en forte croissance ne sont pas suffisantes pour élaborer une théorie et ainsi envisager des mesures politiques précises.

Cette étude suédoise vise principalement à élargir ces connaissances en examinant la dynamique des entreprises en forte croissance dans une perspective «macronomique». Cela implique l'étude de :

- leur contribution à l'emploi ;

- leurs affiliations industrielles, leur groupe d'âge et de taille;

- leurs trajectoires (agrégées) de développement;

- leurs stratégies en termes de croissance interne par rapport à la croissance par acquisition ou fusion.

En plus, différentes sections de cet article porteront sur la méthodologie qui est cruciale pour les questions comme celles énumérées ci-dessus.

\section{Méthodologie}

Base de données. Les données ont été obtenues auprès de l'Office national suédois de la statistique (SCB). Leurs registres sont complets au sens où toutes les activités commerciales et légales y sont répertoriées, peu importe leur forme juridique (société individuelle, société en nom collectif ou en commandite simple, société anonyme). Les données viennent de différentes sources comme l'Administration des contributions directes et indirectes et d'enquêtes mandataires. Les registres sont souvent revus et, d'un point de vue international, sont considérés comme étant de très haute qualité. Pour une description plus détaillée des données, se reporter à Davidsson (1997).

Revue internationale P.M.E., vol. 14, $\mathrm{n}^{\text {os }} 3-4,2001$ 
Nous avons choisi d'utiliser les versions annuelles de SCB Centrala Företagsoch Arbetsställeregistret (CFAR, le registre central des entreprises et établissements) comme base pour nos données. Ce registre comprend les informations suivantes sur chaque entreprise : le nom, l'adresse, la forme juridique, la branche industrielle, le nombre d'établissements, le nombre d'employés, la date d'entrée, active / non active et quelques autres variables. Aucune donnée financière n'apparaît dans ce registre.

Pour augmenter encore la qualité et l'utilisation des données pour cette étude, celles du CFAR ont été combinées avec deux registres du SCB : Koncernregistret (le registre des groupes affiliés) et Registret över Utlandsägda Företag (le registre des entreprises affiliées à des sociétés étrangères). Cela nous permet de distinguer les entreprises indépendantes des entreprises faisant partie d'un groupe et d'indiquer si la société mère est localisée à l'intérieur ou à l'extérieur de la Suède.

Unité d' analyse. En principe, trois nivaux d'analyse sont possibles : l'établissement, l'entreprise et le groupe (s'il y a lieu). L'entreprise et le groupe sont tous les deux d'intérêt pour les chercheurs ainsi que pour les politiciens. Toutefois, en se limitant aux seules entreprises, on ne tient pas compte de la croissance par acquisition ou par fusion contre la croissance interne. Dans le premier cas, l'entreprise n'apparaît pas nécessairement comme une entreprise en forte croissance dans une banque de données centrées sur le niveau de l'entreprise. En revanche, si les analyses se limitent au niveau du groupe d'entreprises, les entreprises en forte croissance à l'intérieur de groupes relativement stables ne seront pas détectées. Par conséquent, nous avons choisi le niveau de l'entreprise. Pour chaque année, nous avons des informations indiquant si l'entreprise est indépendante ou si elle fait partie d'un groupe d'entreprises. Si une entreprise appartient majoritairement à une autre entité juridique ou / et contrôle majoritairement une filiale, elle sera considérée comme un membre de ce groupe.

La base des données comprend toutes les entreprises, en novembre 1996, du secteur privé, commercialement actives en Suède et comptant au moins 20 employés. Cela veut dire que nous travaillons avec la population complète et non pas seulement avec un échantillon. Il existe 11748 entreprises dans cette catégorie, dont 233 furent créées en 1996 et ont en conséquence été exclues de cette étude. Des données annuelles pour toutes les entreprises ont été compilées pour la période 1987-1996; 8562 entreprises ont été en activité pendant toute la période. Les entreprises créées pendant cette période qui ne satisfaisaient pas au critère de taille ainsi que les organisations du secteur public qui passaient au secteur privé au cours de la dernière année ont été exclues de même que les entreprises qui se sont dissoutes pendant la période visée. Pour la taille, aucune limite maximale n'a été établie.

Un problème technique associé à l'utilisation du niveau de l'entreprise (plutôt que celui de l'établissement) se pose du fait que les codes d'entreprises peuvent

Revue internationale P.M.E., vol. 14, $\mathrm{n}^{\text {os }} 3-4,2001$ 
changer lorsqu'il y a changement de propriétaire, reclassification de branche industrielle ou relocalisation géographique. Une entreprise active peut donc apparaître dans les registres comme une fermeture ou une création d'entreprise. Les codes d'identification pour les établissements sont relativement moins sensibles à ce genre de changement et en conséquence « vivent plus longtemps » dans les registres. C'est pourquoi nous n'avons pas accepté les codes d'entreprises comme critère pour suivre le développement des entreprises. Plutôt, des constellations d'établissements (et leurs employés) associées au même code d'entreprises sont considérées comme la «même » entreprise s'ils apparaissent ensemble dans la version annuelle suivante dans le registre sous un code différent.

Description. Les entreprises de cette base de données employaient en tout 1,25 million de personnes en 1996 ; cela représente environ $60 \%$ des emplois totaux dans le secteur privé (Davidsson, Lindmark et Olofsson, 1996). Les $40 \%$ restants sont employés dans des entreprises ayant moins de 20 employés. La distribution des emplois se répartit dans les diverses classes, excepté peut-être pour la classe des entreprises de 250-450 employés. Cela est vraisemblablement relié au problème $\mathrm{du}$ «Mittelstand» [position interne], où les entreprises de taille moyenne sont relativement sous-représentées par rapport aux petites ou très grandes entreprises. Il s'agit cependant là d'un problème que la Suède partage avec la majorité des pays européens (Johansson, 1997).

Le secteur industriel le plus important est le secteur manufacturier (traditionnel), comptant plus de $38 \%$ des employés, suivi du «commerce, restauration et hôtellerie » avec $23,6 \%$. Puisque les microentreprises sont exclues, la part attribuée au manufacturier est ici plus grande que dans le secteur privé en général, où la part non manufacturière est plus petite (Davidsson, Lindmark et Olofsson, 1996).

Définition d' une «entreprise à forte croissance » $(E F C)$. Le principal objectif de ce travail est d'examiner la contribution des entreprises en forte croissance à la création d'emplois. Il est donc retenu que les changements absolus en emplois représentent le meilleur critère de performance puisque nous sommes surtout intéressés à établir combien d'emplois sont vraiment créés dans l'économie et non au développement relatif des entreprises ${ }^{1}$. Nous avons défini les EFC comme les $10 \%$ des cas valides (11 748 moins les 233 qui apparaissent seulement pendant la dernière année dans la base de données) qui affichent la moyenne annuelle en croissance absolue d'emplois la plus élevée.

1. On doit noter que si la performance devait être calculée comme un changement relatif, cela aurait un impact important sur les résultats. La corrélation entre changement absolu en emplois et changement relatif pour chaque année varie entre 0,002 et 0,30 , indiquant une relation très modeste. 
Être une PME n'est pas une condition pour faire partie des EFC ; les PME en forte croissance constituent donc un sous-ensemble de toutes les EFC. Cette définition favorise les grandes entreprises. Si nous avions retenu une définition basée sur la croissance totale des emplois pour la période, cette définition aurait favorisé les jeunes entreprises. La moyenne de croissance annuelle pour les entreprises pour la période 1987-1996 était de 0,1. Le 90e percentile, c'est-à-dire le niveau de distinction pour les entreprises à forte croissance, donnait un taux moyen de 7,75 employés par année. Cela veut dire que nous ne retenons pas de taux de croissance extrême pour le groupe EFC. L'échantillon a été divisé en deux groupes : les entreprises à forte croissance et les autres (le groupe témoin).

La taille initiale de l'entreprise n'entre pas dans nos calculs de croissance. Une importante mesure de correction de données que nous avons prise dans ce contexte est de ne retenir que l'emploi de départ après sa création effective. Nos premières analyses nous confirment que c'est là un choix judicieux, car autrement cela donne des résultats très biaisés (Davis, Haltiwanger et Schuh, 1996).

En évaluant la contribution à la création d'emplois de diverses catégories d'entrepreneurs, l'attention est souvent dirigée vers la création d'emplois véritablement nouveaux. Pour assurer une comparabilité maximale avec d'autres études, nous avons choisi d'inclure le changement total en emplois dans nos calculs de croissance et, en conséquence, dans notre définition des EFC. L'étude permet ainsi de distinguer la croissance interne de la croissance par acquisition. Cela est possible en suivant le développement du statut et de la taille de tous les établissements qui sont associés à une entreprise et en les classant dans cinq catégories : originale, par acquisition précédente, par création précédente, acquis cette année et créée cette année. Nous commenterons au fur et à mesure sur la proportion des emplois créés qui est ou non interne. Pour cela, nous avons calculé la croissance interne annuelle de la manière suivante: emplois totaux $_{(t)}$ - emplois totaux $(t-1)-$ emplois dans les établissements acquis l'année précédente - emplois dans les établissements acquis cette année. Il est important de remarquer que le développement dans les unités acquises au cours des années précédentes est inclus dans la croissance interne de l'entreprise.

Choix de variables de description. Quatre variables furent utilisées pour décomposer l'analyse en sous-catégories : la taille de l'entreprise (six classes), la branche industrielle ( 16 branches), l'âge (nombre d'années dans les registres) et la gouvernance (c'est-à-dire indépendant ou affilié à un groupe comme filiale ou siège social). Dans la plupart des analyses, nous comprimons les données par catégories plus agrégées que celles indiquées entre parenthèses, par exemple entre petites et grandes entreprises.

La taille de l'entreprise a été choisie à cause de son importance supposée pour la croissance et la création d'emplois (Dunne et Hughes, 1996; Storey, 1995 ;

Revue internationale P.M.E., vol. 14, $\mathrm{n}^{\text {os }}$ 3-4, 2001 
Wagner, 1992). La branche industrielle a été retenue pour deux raisons : premièrement, la majorité des recherches sur la croissance ont porté sur des échantillons ne comprenant que le secteur manufacturier (Delmar, 1997); deuxièmement, l'importance du secteur des services en tant que créateur d'emplois a considérablement augmenté pendant les dernières décennies. L'âge est une variable qui revient dans la plupart des études sur la croissance des entreprises; normalement, les jeunes entreprises sont plus propices à grandir que les entreprises plus âgées et bien établies (Evans, 1987). L'âge est aussi fortement associé aux possibilités de survie d'une entreprise : une jeune entreprise qui crôt double sa probabilité de survie par rapport à une jeune entreprise qui ne croît pas (Phillips et Kirchhoff, 1989). Diverses hypothèses peuvent aussi être émises en ce qui a trait à la gouvernance. Si une entreprise indépendante peut jouir d'une grande flexibilité et d'une marge de manœuvre considérable, celle qui est affiliée à un groupe peut probablement disposer d'un accès important à certaines ressources.

Dans l'interprétation des résultats qui suivent, il importe de retenir que le groupe des EFC et le groupe témoin représentent des «cibles mouvantes », car chaque année, entre 1987 et 1996, de nouveaux cas apparaissent dans les deux catégories. Ainsi, dans les données de 1987-1988, l'échantillon comprenait 8562 entreprises, tandis que dans celles de 1995-1996, il y avait 11515 entreprises. Les groupes EFC et témoin sont stables puisque peu importe la performance d'une entreprise pendant une année donnée, cette entreprise fera partie soit des EFC, soit du groupe témoin selon sa performance totale durant toute la période étudiée.

\section{Résultats}

\subsection{L'affiliation par catégories des entreprises en forte croissance}

Une première question d'importance est la suivante : comment les EFC sont distribuées dans les classes de taille, les branches industrielles, etc., en comparaison des entreprises du groupe témoin? Le tableau 1 donne les résultats en pourcentage d'entreprises qui sont associées aux diverses catégories en 1996.

En ce qui concerne les classes de taille, nous observons que les EFC sont sousreprésentées dans la classe de petite taille, mais surreprésentées dans la classe des 50-249 employés, c'est-à-dire les entreprises de taille moyenne. Pendant que $76 \%$ des EFC sont (encore) des PME (ayant moins de 250 employés), le groupe des EFC affiche une surreprésentation des grandes entreprises (ayant plus de 250 employés). Cela semble aller à l'encontre de l'opinion répandue voulant que les PME sont les plus importants créateurs d'emplois. Or, ce résultat est en partie dû à notre définition des EFC ; une définition basée sur la croissance relative aurait favorisé les entreprises plus petites. En général, cette surreprésentation des grandes 
EFC résulte simplement de leur croissance. Parmi les $24 \%$ qui étaient grandes en 1996, près de la moitié d'entre elles étaient considérées auparavant comme PME. Donc, 87,6\% des EFC étaient des PME à un moment donné au cours de la période 1987-1996.

TABLEAU 1

Distribution des EFC dans les diverses catégories 1996 (1995)

\begin{tabular}{|c|c|c|}
\hline & $\begin{array}{l}\text { Entreprises à } \\
\text { forte croissance } \\
\quad(n=1153)\end{array}$ & $\begin{array}{l}\text { Groupe témoin } \\
\qquad(n=10595)\end{array}$ \\
\hline Taille (nombre d'employés) & $\%$ & $\%$ \\
\hline $20-49$ & 29,7 & 68,2 \\
\hline $50-249$ & 46,1 & 27,6 \\
\hline $250-499$ & 11,0 & 2,3 \\
\hline $500-2499$ & 11,0 & 1,7 \\
\hline 2500 et plus & 2,2 & 0,2 \\
\hline \multicolumn{3}{|l|}{ Industries } \\
\hline Haute technologie & 4,9 & 3,1 \\
\hline Bois, pâtes et papier & 2,9 & 5,9 \\
\hline Industries d'ingénierie & 8,8 & 13,8 \\
\hline Mines et acier & 1,1 & 2,0 \\
\hline Autres entreprises manufacturières & 7,4 & 11,5 \\
\hline Services à forte connaissance - technique & 10,2 & 4,6 \\
\hline Services à forte connaissance - financier & 6,0 & 3,6 \\
\hline Services à forte connaissance - autres & 5,9 & 3,1 \\
\hline Construction & 6,8 & 9,0 \\
\hline Gros et détail & 16,7 & 24,5 \\
\hline Hôtels et restaurants, etc. & 6,5 & 4,8 \\
\hline Transport & 7,4 & 6,5 \\
\hline Éducation et soins de santé & 10,2 & 3,9 \\
\hline Personnel et services sociaux & 5,0 & 2,7 \\
\hline Foresterie, agriculture et pêche & 0,3 & 1,0 \\
\hline \multicolumn{3}{|l|}{$\overline{\hat{A} g e}$} \\
\hline Pérennes durant toute la période 1987-1996 & 37,9 & 73,0 \\
\hline Créées pendant la période & 62,1 & 27,0 \\
\hline \multicolumn{3}{|l|}{ Affiliées à un groupe $(1995)^{*}$} \\
\hline Indépendantes & 35,0 & 38,3 \\
\hline Parentes & 12,7 & 14,5 \\
\hline Filiales & 52,3 & 47,2 \\
\hline
\end{tabular}

* Les données pour la gouvernance qui n'étaient pas disponibles en 1996 ont été remplacées par des données de 1995 . 
En ce qui concerne les branches industrielles, nous observons que les EFC sont représentées dans toutes les branches. Mais il existe une surreprésentation dans les industries jeunes et en expansion et une sous-représentation dans les branches traditionnelles. La surreprésentation est particulièrement prononcée dans les secteurs de services à forte connaissance, l'éducation et les soins de santé (Davidsson, Lindmark et Olofsson, 1996). Blixt (1997) et Storey (1996), qui ont étudié la situation en Suède et en Grande-Bretagne respectivement, utilisent tous deux la croissance du chiffre d'affaires comme indicateur de performance dans leurs définitions d'entreprises à forte croissance et obtiennent par conséquent des résultats différents. Mais l'impression générale que l'occurrence des EFC est reliée à la croissance relative de la branche industrielle est aussi relevée dans leurs études.

Pour ce qui est de l'âge, il existe une large surreprésentation chez les EFC des entreprises créées pendant la période à l'étude, et ce, bien que nous ayons pris des précautions méthodologiques pour ne pas favoriser les jeunes entreprises. Le message est clair: les EFC se retrouvent surtout parmi les jeunes entreprises. Blixt (1997) et Storey (1996) trouvent aussi une nette surreprésentation de jeunes entreprises parmi les EFC et la même relation âge-croissance a aussi été relevée dans plusieurs autres études (voir par exemple Storey, 1994 ; Evans, 1987).

Dans les dernières rangées du tableau, on voit que, même si la différence entre les groupes est minime à cet égard, une majorité d'entreprises dans les deux groupes est affiliée à un groupe. Signalons que les filiales EFC peuvent faire partie d'un groupe qui, lui, n'est pas considéré comme en «forte croissance ». Suivant la même logique, quelques-unes des sociétés mères figurant dans le groupe témoin peuvent diriger des firmes que nous pourrions considérer «en forte croissance». Pourtant, il faut savoir que $56 \%$ des EFC et $64 \%$ des entreprises du groupe témoin ont déjà été indépendantes. Ni Blixt (1997) ni Storey (1996) ne rapportent des résultats comparables au sujet de la gouvernance.

En somme, si la plupart des EFC sont des PME, près des deux tiers sont affiliées à des groupes d'entreprises qui, dans certains cas, forment ensemble des «grandes entreprises ». Elles sont remarquablement plus jeunes que celles du groupe témoin et, quoique présentes dans toutes les branches, elles sont nettement surreprésentées dans les branches jeunes et dans les services en expansion.

\subsection{La contribution des entreprises en forte croissance à l'emploi}

Dans cette section, nous analysons la contribution totale des EFC à la création d'emplois ainsi que par taille, branches industrielles et type de gouvernance. Dans chaque analyse, nous donnons la contribution des EFC et des firmes du groupe témoin pour la dernière année de l'analyse, soit de novembre 1995 à novembre 1996. 
Le tableau est accompagné d'une figure décrivant le développement pour l'ensemble de la période à l'étude, 1987-1996. Dans cette figure, l'affiliation par catégorie est basée sur les données de l'année en question, sauf l'appartenance aux EFC ou au groupe témoin ; les entreprises peuvent toutefois changer de catégorie d'une année à l'autre. La question de la croissance interne ou par acquisition sera traitée dans la prochaine section.

En interprétant les données, il est important de s'arrêter au nombre d'entreprises incluses dans l'analyse; le nombre des EFC est toujours inférieur à celui des entreprises du groupe témoin. De plus, en passant de 1996 à 1987, le nombre d'entreprises diminue plus dans les EFC que dans le groupe témoin (puisque plus de EFC furent créées durant cette période, voir tableau 1 et annexe I).

Le tableau 2 illustre le changement en emplois absolus pour les EFC et les firmes du groupe témoin en 1995-1996. Comme on peut le voir, les deux groupes affichent une création d'emplois nette pour cette période. On notera que le groupe des $\mathrm{EFC}$ (beaucoup plus petit en nombre) crée près de $80 \%$ des nouveaux emplois, même si l'on ne lui attribue qu'un peu plus de $30 \%$ des emplois totaux. Mais les EFC contribuent-elles à une large partie des nouveaux emplois en termes absolus? Dans un sens oui, puisque ces firmes créent en moyenne 40 nouveaux emplois par entreprise, bien que ces 45000 nouveaux emplois restent un chiffre faible en comparaison des centaines de milliers de personnes sans emploi. En outre, ce chiffre total est du même ordre que celui associé à la création d'entreprises chaque année en Suède (Statistics Sweden, 1995, 1997). Selon une autre source, la création et l'expansion de petites entreprises, ayant entre 0-200 employés, ont contribué à 70000 nouveaux emplois en 1994 (Davidsson, Lindmark et Olofsson, 1996). Une grande partie de ces emplois est attribuable aux entreprises ayant moins de 20 employés et qui sont exclues de notre analyse.

Considérant que nous avons utilisé une limite plutôt permissive pour les EFC (7,75 nouveaux emplois par an en moyenne) et que 1995-1996 représente leur «meilleure année » (figure 1), nous pouvons conclure que les EFC telles qu'elles ont été définies constituent une source importante de nouveaux emplois, mais ne surpassent pas d'autres catégories de créateurs d'emplois comme les très petites entreprises nouvellement créées et en expansion.

Sur le plan méthodologique, le résultat le plus important de la figure 1 est que le groupe des EFC, en dépit de leur faible nombre et de la variation conjoncturelle, performe mieux que le groupe témoin chaque année. Cela est d'autant plus impressionnant que le nombre de cas diminue plus rapidement pour le groupe des EFC que pour le groupe témoin lorsque nous revenons de 1996 en arrière $\left(n_{1987}=437\right.$ entreprises et 7736 emplois; voir le tableau A1). Signalons en outre que la différence de performance est plus prononcée durant les pires années de la récession, soit 1992 et 1993 ; les EFC ont apparemment été moins affectées par les 
changements dans l'économie générale. En effet, elles ont eu tendance à demeurer relativement stables pendant que l'ensemble des autres entreprises perdait un grand nombre d'emplois. Nous reviendrons là-dessus à la fin de ce travail.

La croissance cumulative totale des emplois par les EFC pour toute la période était de 185000 , ce qui est un chiffre imposant, mais qui est loin de compenser pour les 255633 emplois nets disparus dans les entreprises du groupe témoin (sans compter tous les emplois perdus par fermeture et non considérés dans ces données ; Davidsson, Lindmark et Olofsson, 1996). Nous pouvons donc conclure que les entreprises à forte croissance ont un effet - bien que insuffisant - sur l'emploi.

TABLEAU 2

Changement dans l'emploi chez les EFC et les entreprises du groupe témoin, 1995-1996

\begin{tabular}{lccccc}
\hline & Changement & Fréquence & Emplois & Fréquence & $\begin{array}{c}\text { Nombre } \\
\text { d'entreprises }\end{array}$ \\
\hline $\begin{array}{l}\text { Entreprises à } \\
\text { forte croissance }\end{array}$ & 45294 & $79,7 \%$ & 400917 & $32,0 \%$ & 1153 \\
\hline Groupe témoin & 11537 & $20,3 \%$ & 851498 & $68,0 \%$ & 10362 \\
\hline Total & 56831 & $100 \%$ & 1252415 & $100 \%$ & 11515 \\
\hline
\end{tabular}

FIGURE 1

\section{Changement dans l'emploi total par groupe de performance, 1987-1996}

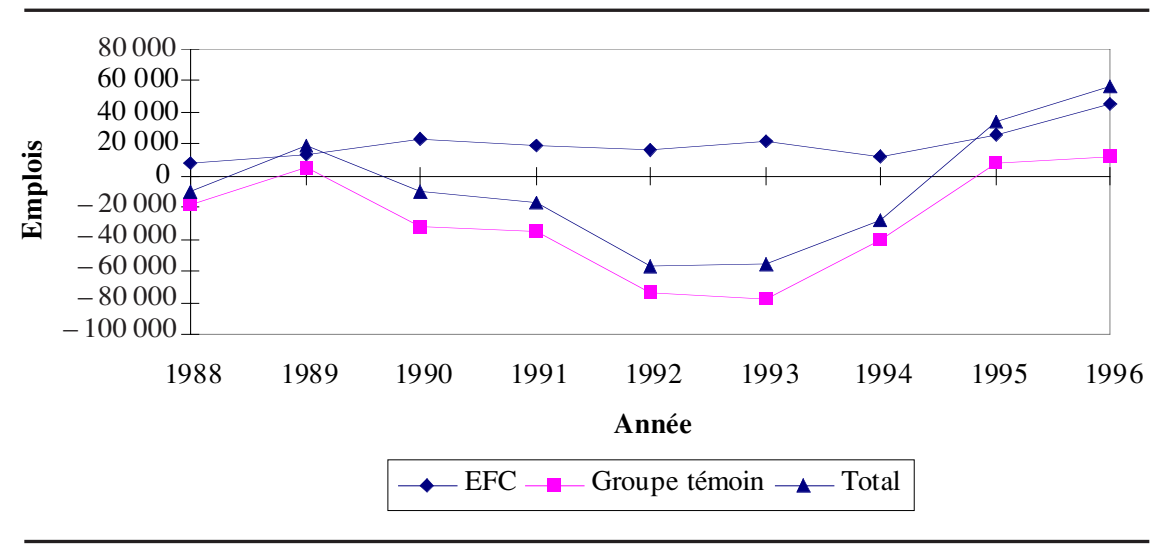

Nous allons maintenant porter notre attention sur la contribution à l'emploi des EFC par sous-catégories. La classification la plus intéressante semble être celle relative à la taille. Le tableau 3 montre les changements absolus dans l'emploi pour chaque taille d'entreprise en 1995-1996. Ensemble, les petites et moyennes 
entreprises (ayant moins de 250 employés) contribuent à la majorité des emplois (53,8 \% en emplois nets). Cependant, parmi les EFC, les grandes entreprises sont les grandes créatrices d'emplois, alors que dans le groupe témoin les grandes entreprises perdent la majorité des emplois. Ce résultat est confirmé pour toute la période analysée (voir figure 2).

Nous pouvons facilement observer les différences entre les deux classes de taille, alors que les petites et moyennes entreprises sont constamment plus performantes que les grandes entreprises. Pendant les années de récession, les grandes entreprises perdent un nombre très important d'emplois. Autrement dit, les grandes entreprises affichent une plus grande variance en performance que les petites et moyennes entreprises pendant cette période.

TABLEAU 3

Changement dans l'emploi par taille d'entreprise en pourcentage, 1995-1996

\begin{tabular}{lccc}
\hline & EFC & Groupe de contrôle & Total \\
\hline Entreprises $<250$ employés & $13867(30,6 \%)$ & $16735(145,1 \%)$ & $\begin{array}{c}30602(53,8 \%) \\
n=9929\end{array}$ \\
& $n=874$ & $n=10803$ \\
\hline Entreprises $>250$ employés & $31427(69,4 \%)$ & $-5198(-45,1 \%)$ & $26229(46,1 \%)$ \\
& $n=279$ & $n=433$ & $n=712$ \\
\hline Total $(n=11515)$ & $45294(100 \%)$ & $11537(100 \%)$ & $56831(100 \%)$ \\
\hline
\end{tabular}

FIGURE 2

\section{Changement dans l'emploi absolu par classe de taille, 1987-1996}

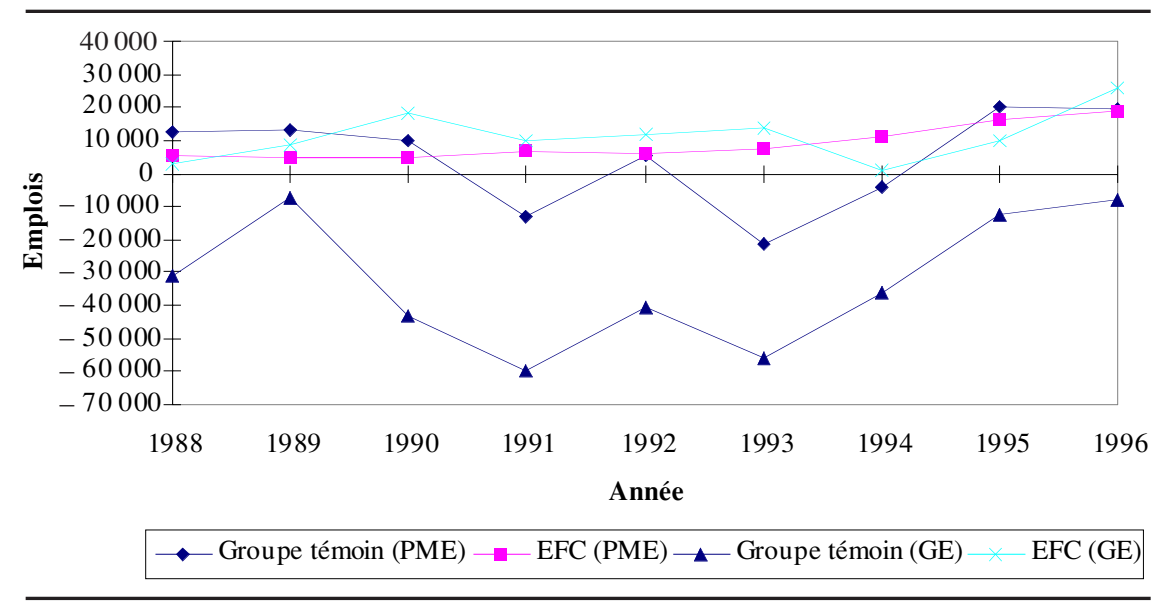

Revue internationale P.M.E., vol. 14, $\mathrm{n}^{\text {os }}$ 3-4, 2001 
Au tableau 4, nous pouvons voir les changements dans l'emploi et leur distribution par secteur industriel pour 1995-1996. Les EFC dans le secteur des services créent près de $60 \%$ de tous les nouveaux emplois; cela souligne encore une fois l'importance croissante du secteur des services pour la création d'emplois (voir la figure 3). Les entreprises à forte croissance dans le secteur des services dépassent constamment le développement des autres groupes. Au cours de la même période, le groupe témoin composé d'entreprises du secteur manufacturier affiche un déclin dramatique sauf dans les dernières années. Bref, le secteur des services a le développement le plus positif et il est largement dû aux EFC qui créent la majorité des emplois.

TABLEAU 4

Changement dans l'emploi et leur distribution dans les grands secteurs industriels en pourcentage, 1995-1996

\begin{tabular}{lccc}
\hline Branche & $\begin{array}{c}\text { Entreprises } \\
\text { à forte croissance }\end{array}$ & Groupe témoin & Total \\
\hline Manufacture & $11213(24,8 \%)$ & $8425(73,0 \%)$ & $9638(34,6 \%)$ \\
& $n=288$ & $n=3783$ & $n=4071$ \\
\hline Service & $34081(75,2 \%)$ & $3112(27,0 \%)$ & $37193(65,4 \%)$ \\
& $n=865$ & $n=6579$ & $n=7444$ \\
\hline Total $(n=11515)$ & $45294(100 \%)$ & $11537(100 \%)$ & $56831(100 \%)$ \\
\hline
\end{tabular}

FiguRE 3

Changements dans l'emploi par secteur industriel, 1987-1996

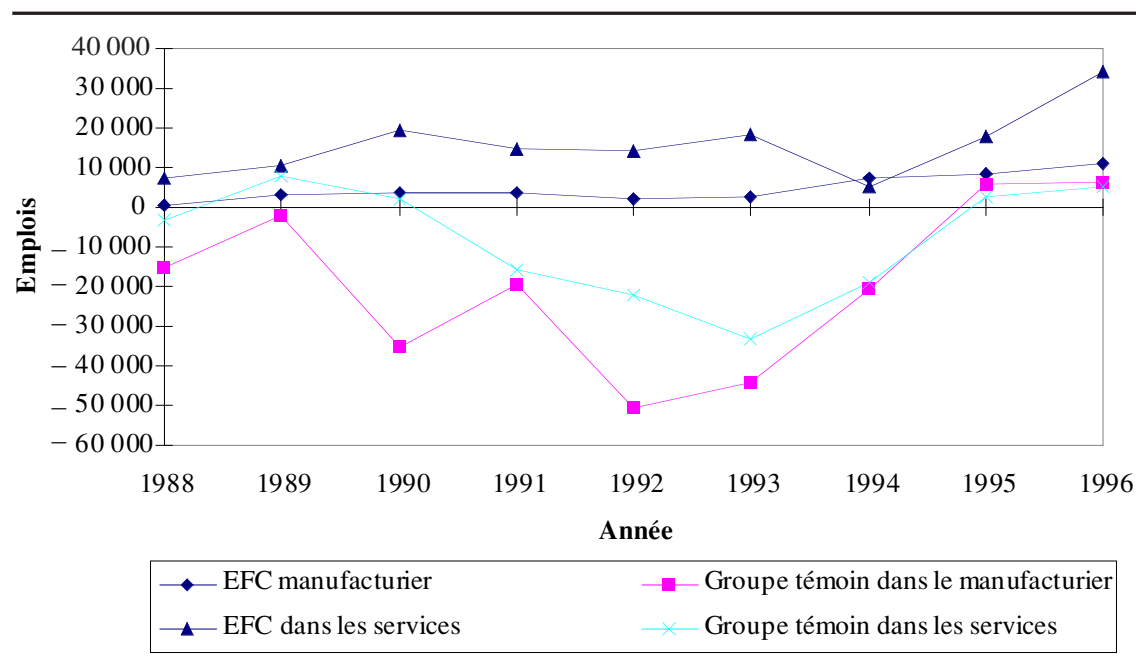


Suivant le tableau 1, la majorité des EFC étaient, à la fin de la période étudiée, affiliées à un groupe d'entreprises; ce qui est confirmé dans notre analyse des changements dans l'emploi. En effet, les EFC faisaient partie d'un groupe créant plus d'emplois que les autres, soit $60 \%$ de tous les nouveaux emplois pendant la période 1995-1996. La différence entre les EFCs indépendantes et les autres entreprises indépendantes du groupe témoin est moins importante. Aussi, la moyenne de croissance est plus élevée pour les EFC affiliées que pour leurs homologues indépendantes. Les changements en emplois absolus par type d'organisation sont montrés au tableau 5.

Ces résultats sont stables pendant toute la période sous étude (voir figure 4) : les entreprises faisant partie d'un groupe sont moins performantes que les entreprises indépendantes, ce qui est conforme aux résultats obtenus auparavant par Davidsson, Lindmark et Olofsson (1997a, 1997b). Signalons que les EFC faisant partie d'un groupe performent mieux que tout autre entreprise, et ce chaque année à l'exception de 1993. Mais la variance dans le changement des emplois est beaucoup plus grande chez les entreprises faisant partie d'un groupe que chez les indépendantes. Les entreprises à forte croissance appartenant à un groupe créent beaucoup d'emplois, mais celles de cette sous-catégorie qui font partie du groupe témoin en créent encore plus.

TABLEAU 5

Changement dans l'emploi par type de gouvernance (indépendant ou partie d'un groupe) en pourcentage, 1995-1996

\begin{tabular}{lccc}
\hline & $\begin{array}{c}\text { Entreprises } \\
\text { à forte croissance }\end{array}$ & Groupe témoin & Total \\
\hline Entreprises indépendantes & $11093(24,5 \%)$ & $7579(65,7 \%)$ & $18672(32,9 \%)$ \\
& $n=404$ & $n=3961$ & $n=4365$ \\
\hline Affiliées à un groupe & $34201(75,5 \%)$ & $3958(34,3 \%)$ & $38159(67,9 \%)$ \\
& $n=749$ & $n=6401$ & $n=7150$ \\
\hline Total $(n=11515)$ & $45294(100 \%)$ & $11537(100 \%)$ & $56831(100 \%)$ \\
\hline
\end{tabular}

Pour conclure cette section, nous relevons que les entreprises à forte croissance représentent certes une source importante de nouveaux emplois, mais, comme nous l'avons déjà mentionné, cette catégorie n'a pas créé d'emplois à un rythme qui impressionne si l'on songe au taux de chômage. En chiffre absolu, leur contribution n'est pas beaucoup plus grande que celle des entreprises récemment créées. Un aspect qui attire particulièrement l'attention est le comportement des EFC pendant la récession; elles semblent être à contre-courant et contrebalancent en conséquence l'effet de la récession sur la main-d'œuvre.

Revue internationale P.M.E., vol. 14, nºs 3-4, 2001 
FigURE 4

Changements dans l'emploi absolu par type de gouvernance (indépendant ou partie d'un groupe), 1987-96

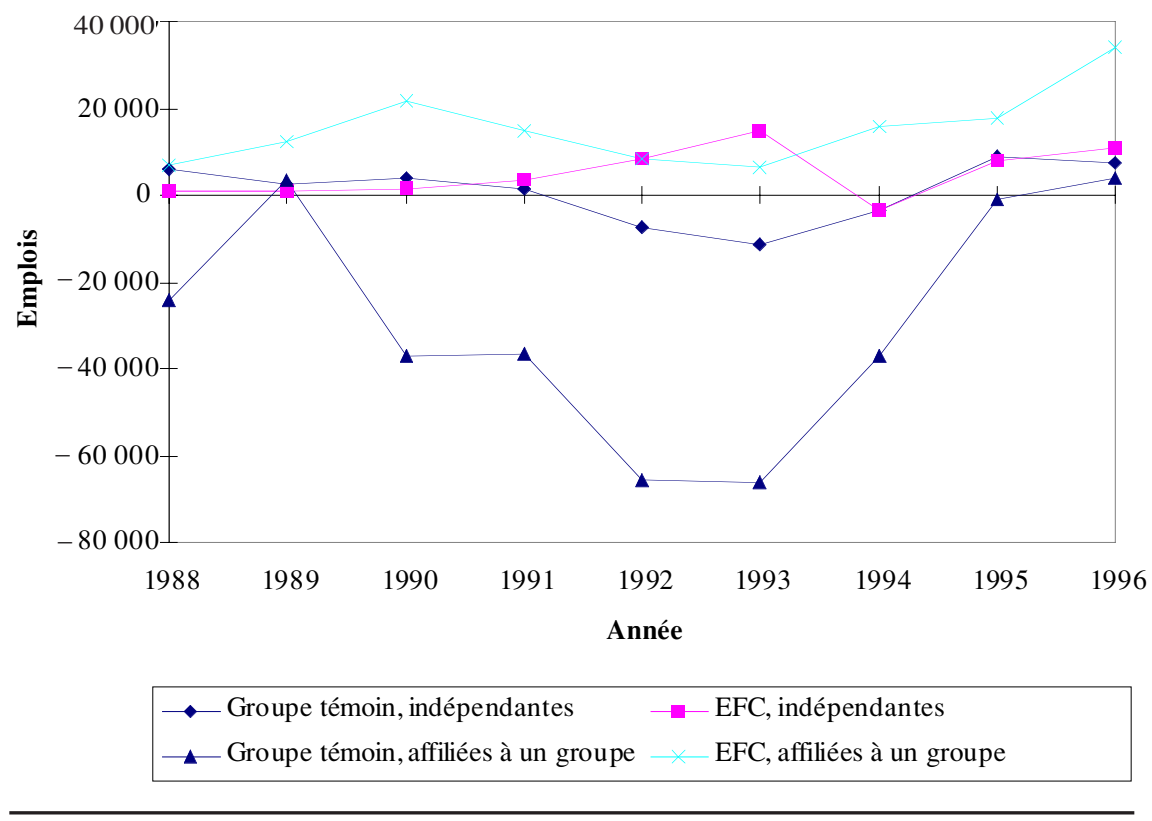

\subsection{Un autre regard sur la création interne d'emplois dans les entreprises à forte croissance}

Notre intérêt réel concerne toutefois les vrais nouveaux emplois, c'est-à-dire les changements dans l'emploi qui affectent le taux de chômage. Pour cette raison, nous allons dans cette section continuer à discuter de ce sujet à l'intérieur du groupe des EFC dans le but d'examiner ce qui change dans les résultats rapportés auparavant quand la croissance interne est utilisée comme critère.

Que la contribution à la création d'emplois par les EFC soit modeste est un résultat qui est renforcé si nous faisons abstraction de la croissance par acquisition. La croissance cumulative interne pour les EFC pour toute la période à l'étude est de 60040 nouveaux emplois, ce qui veut dire que moins d'un tiers de la croissance des EFC était interne. Si nous prenons les EFC par branche industrielle en 1996, nous voyons que $21,4 \%$ des EFC du secteur manufacturier ont crû de façon interne. Le chiffre correspondant pour les EFC du secteur des services est de 37,3\%. Ainsi, le poids relatif du secteur des services augmente si c'est la croissance interne qui 
est utilisée comme critère plutôt que la croissance totale. Des analyses plus détaillées des affiliations industrielles révèlent que la croissance interne est plus importante dans les branches jeunes et en croissance.

En ce qui concerne la gouvernance, nous relevons des différences notables entre les catégories. Pour les EFC indépendantes en 1996, la croissance cumulative interne représente $64,5 \%$ de leur croissance totale. Chez les EFC affiliées à des groupes, la part de croissance interne est inférieure $(25,3 \%)$. En termes absolus, cela signifie que la croissance interne cumulative des EFC appartenant à des groupes dépasse encore la croissance interne des EFC indépendantes (38 369 contre 21 671); mais la relation est bien différente si l'on compare la croissance cumulative totale (151 684 par rapport à 33580 ).

TABLEAU 6

Croissance totale et interne pour EFC selon l'ancienneté

\begin{tabular}{ccccc}
\hline $\begin{array}{c}\text { Nombre d'années } \\
\text { en existence dans } \\
\text { la base de } \\
\text { données }\end{array}$ & $\begin{array}{c}\text { Nombre de cas } \\
(\boldsymbol{n})\end{array}$ & $\begin{array}{c}\text { Croissance } \\
\text { cumulative totale } \\
\text { des emplois }\end{array}$ & $\begin{array}{c}\text { Croissance } \\
\text { cumulative interne } \\
\text { des emplois }\end{array}$ & $\begin{array}{c}\text { Croissance } \\
\text { interne } \\
(\%)\end{array}$ \\
\hline 2 & 148 & 3319 & 3191 & 96,1 \\
3 & 205 & 8865 & 7052 & 79,5 \\
4 & 137 & 6984 & 6118 & 87,6 \\
5 & 77 & 7043 & 6619 & 94,0 \\
6 & 40 & 3912 & 3429 & 87,7 \\
7 & 42 & 6364 & 4401 & 69,2 \\
8 & 38 & 3920 & 2992 & 76,3 \\
9 & 29 & 6919 & 4038 & 58,4 \\
10 & 437 & 137938 & 22200 & 16,1 \\
\hline Total & 1153 & 185264 & 60040 & 32,4 \\
\hline
\end{tabular}

Ces analyses montrent jusqu'à quel point de fausses conclusions peuvent être tirées si l'on néglige de distinguer croissance interne et croissance par acquisition. Cette distinction est particulièrement importante au regard de l'âge et de la taille de l'entreprise. Dans le tableau 7, on voit que la part de la croissance totale interne est beaucoup plus importante chez les EFC créées pendant la période étudiée que chez les EFC déjà en activité en 1987. Évidemment, pour les entreprises plus anciennes, une bonne part de « la création d'emplois » représente en réalité une restructuration; ce changement d'optique est important. En utilisant le critère de croissance totale, près des trois quarts de la croissance en emplois des EFC sont attribuables à des entreprises plus anciennes et plus établies, alors que leur part de la croissance à l'interne est inférieure à un tiers. Le message est clair : plus l'entreprise est jeune, plus sa croissance totale se réalisera à l'interne.

Revue internationale P.M.E., vol. 14, nos 3-4, 2001 
Traitons maintenant de la taille des entreprises. Deux analyses sont présentées au tableau 7, soit la croissance totale et à l'interne par taille initiale pour les EFC pérennes pendant toute la période 1987-1996. Puisque la croissance est par définition ce qui fait grandir les PME, il est avantageux d'examiner leur taille initiale pour analyser de quelle classe de taille les nouveaux emplois sont originaires. Toutefois, cette analyse n'utilise qu'une partie de l'échantillon et toute la croissance peut être attribuée à la classe de (petite) taille initiale même si la plus grande partie de la croissance a lieu après que l'entreprise est passée à une classe de taille plus grande. C'est pourquoi nous avons complété cette analyse par une seconde qui se rapporte à la croissance à l'interne et totale pour toutes les EFC selon leur classe de taille en 1996.

TABLEAU 7

\section{Croissance totale et à l'interne pour les EFC de tailles différentes}

\begin{tabular}{lcccc}
\hline $\begin{array}{c}\text { Classe de taille } \\
\mathbf{1 9 8 7}\end{array}$ & $\begin{array}{c}\text { Nombre de cas } \\
(\boldsymbol{n})\end{array}$ & $\begin{array}{c}\text { Croissance } \\
\text { cumulative totale } \\
\text { des emplois }\end{array}$ & $\begin{array}{c}\text { Croissance cumulative } \\
\text { à l'interne des emplois }\end{array}$ & $\begin{array}{c}\text { Croissance } \\
\text { à l'interne } \\
(\boldsymbol{\%})\end{array}$ \\
\hline 0 & 30 & 6088 & 4897 & 80,4 \\
$1-9$ & 35 & 4461 & 4182 & 93,7 \\
$10-49$ & 91 & 11617 & 7797 & 67,1 \\
$50-249$ & 188 & 32705 & 17422 & 53,2 \\
$250-499$ & 37 & 11913 & 2339 & 19,6 \\
$500-2499$ & 73 & 50492 & 3542 & 7,0 \\
$2500+$ & 13 & 26750 & -13082 & $(-48,9)$ \\
\hline Total & 467 & 144026 & 27097 & 18,8 \\
\hline
\end{tabular}

Croissance totale et à l'interne pour les EFC de tailles différentes

\begin{tabular}{lcccc}
\hline $\begin{array}{c}\text { Classe de taille } \\
\mathbf{1 9 9 6}\end{array}$ & $\begin{array}{c}\text { Nombre de cas } \\
(\boldsymbol{n})\end{array}$ & $\begin{array}{c}\text { Croissance } \\
\text { cumulative totale } \\
\text { des emplois }\end{array}$ & $\begin{array}{c}\text { Croissance cumulative } \\
\text { à l'interne } \\
\text { des emplois }\end{array}$ & $\begin{array}{c}\text { Croissance } \\
\text { à l'interne } \\
(\%)\end{array}$ \\
\hline $20-49$ & 342 & 8124 & 7963 & 98,0 \\
$50-249$ & 532 & 44320 & 34208 & 77,2 \\
$250-499$ & 127 & 22340 & 12497 & 55,9 \\
$500-2499$ & 127 & 57752 & 15682 & 27,2 \\
$2500+$ & 25 & 52728 & -10310 & $(-19,6)$ \\
\hline Total & 1153 & 185264 & 60040 & 32,4 \\
\hline
\end{tabular}

À ce sujet, les deux analyses convergent : plus l'entreprise est petite, plus la part de la croissance à l'interne sur la croissance totale est grande. Ce résultat est encore plus évident qu'avec la variable âge de l'entreprise, puisque les deux analyses montrent que les EFC ayant plus de 2500 employés décroissent à l'interne. Cela est peut-être notre résultat le plus étonnant: si ce sont les «vrais » emplois que nous recherchons, l'analyse de la croissance totale nous mène à des résultats tout à fait erronés. 
La classe de taille des EFC qui dominerait pour la création d'emplois serait celle des 50-249 employés. Cela est plutôt ironique, car, selon l'opinion générale, ce qui manque en Suède, ce sont justement les entreprises de taille moyenne en croissance. Nos résultats révèlent que, parmi les entreprises qui ont atteint ce niveau, nous trouvons aussi un groupe important d'EFC dont la croissance est en majorité interne. Or, le nombre total d'emplois créés par les entreprises de taille moyenne est faible en comparaison des taux de chômage; d'un point de vue des politiques, on voudrait certes avoir un plus grand nombre de ces entreprises. C'est plutôt ici que réside le véritable problème. Pendant que le nombre absolu d'entreprises dans l'économie en général est beaucoup plus grand dans la classe des moins de 50 employés que dans la classe des 50 à 249 employés, le nombre de EFC dans la première classe est plus faible. Même si, pour la plupart, la croissance des emplois est interne, cela ne change pas le fait qu'il y a trop peu d'EFC dans cette classe de taille.

En somme, les analyses portant sur la croissance interne montrent que certaines catégories d'EFC sont plus importantes pour la création de « vrais » emplois que les analyses sur la croissance totale ne le laissent entendre. Les entreprises jeunes, petites, œuvrant dans des branches jeunes et en croissance prennent beaucoup plus de poids lorsque nos analyses excluent les acquisitions qui, apparemment, procèdent à des restructurations et ne créent pas de nouveaux emplois.

\section{Conclusion}

\section{Principaux résultats}

Pour mener cette étude, nous avons considéré les entreprises à forte croissance dans le secteur privé en Suède pendant la période 1987-1996 en utilisant une banque de données de très haute qualité qui a été mise sur pied dans cette optique. Nous avons restreint notre étude au niveau de l'entreprise et aux entreprises ayant au moins 20 employés au cours de la dernière année, soit en 1996. Comme notre principal intérêt est la création d'emplois, nous avons défini les entreprises à forte croissance comme les $10 \%$ qui avaient le plus contribué à l'emploi en croissance moyenne absolue par an. Pour faire partie de ce groupe, une entreprise devait afficher une croissance minimale de 7,75 employés par an. Cela peut aussi être considéré comme un premier résultat: faire partie de ces EFC ne demande pas une performance extrême en Suède pour la période visée. C'est au cours de la dernière année d'observation que la contribution est la plus élevée (1995-1996). Pendant cette année de conjoncture favorable, les EFC ont créé près de 40 emplois en moyenne par entreprise. Cependant, même agrégé, ce chiffre ne semble pas impressionnant. Cette contribution est comparable à celle qu'apportent annuellement les entreprises nouvellement créées, et même un doublement de la contribution des entreprises à forte croissance ne saurait réduire de façon satisfaisante le taux de chômage.

Revue internationale P.M.E., vol. 14, $\mathrm{n}^{\text {os }} 3-4,2001$ 
Nous avons relevé une surreprésentation des EFC dans certaines souscatégories. En particulier, les jeunes entreprises ont beaucoup plus de chances d'être en forte croissance que les plus âgées. Nous avons également relevé une surreprésentation dans les branches industrielles jeunes et en croissance, en particulier les services à fortes connaissances, l'éducation et les soins de santé. Une légère majorité des $\mathrm{EFC}$ sont affiliées à des groupes et $13 \%$ constituent la société mère du groupe. Ces résultats concernent la croissance totale des emplois au niveau de l'entreprise.

Lorsqu'on s'intéresse exclusivement à la croissance à l'interne, certains de nos résultats sont renforcés, alors que d'autres changent. Les changements les plus importants concernent l'âge et la taille de l'entreprise. Chez les EFC âgées de cinq ans ou moins, $80 \%$ ou plus de la croissance est interne et censée refléter une création d'emplois véritable. En revanche, seulement $16 \%$ de la croissance des EFC de dix ans ou plus est interne. Pour la taille d'entreprise, l'effet est encore plus dramatique; les EFC les plus grandes perdent des emplois à l'interne.

L'impression générale qui se dégage de nos données est que les entreprises jeunes et petites, souvent dans des branches jeunes et en expansion, sont en grande partie responsables de la création de « vrais » emplois, c'est-à-dire des emplois qui ont un effet direct sur les taux de chômage. Les grandes entreprises établies, œuvrant souvent dans des branches matures, sont responsables de plus de changement, mais le chiffre net a tendance à être négatif pour ce qui est du nombre d'emplois. Cette dernière catégorie comprend toutefois un groupe d'entreprises en croissance qui assure une part relativement large de la création nette d'emplois ; à notre avis, cela semble résulter de restructurations plutôt que d'une création véritable d'emplois.

Vraisemblablement, lorsque les entreprises sont jeunes et petites, si elles prennent de l'expansion, c'est à travers une croissance interne. En prenant de la maturité, elles sont plus portées à acquérir des activités existantes en fermant ou vendant leurs unités obsolètes. Ce qui est important, c'est que les deux rôles sont centraux au bon développement de l'économie : la croissance interne à travers de nouvelles activités, où les entreprises jeunes et petites jouent un rôle important, et la restructuration pour une utilisation plus effective des ressources, où les grandes entreprises dominent.

\section{Implications}

L'un de nos résultats saillants est le manque d'entreprises à forte croissance en Suède (ou du moins pendant la période 1987-1996). Cela justifie un examen des cadres institutionnels contrôlés politiquement et de leurs effets sur le nombre

d'entreprises à forte croissance et leur capacité à atteindre une croissance rapide (Davidsson et Henrekson, à paraître). 
Nos analyses montrent que les EFC, quoique surreprésentées dans les branches jeunes et en expansion, apparaissent aussi dans des branches stagnantes ou en déclin. Cela laisse croire qu'il existe des possibilités pour les politiques de choisir. Pour les entrepreneurs, la leçon à retenir, c'est que des niches de croissance peuvent être trouvées même dans des contextes difficiles. Un résultat frappant de notre analyse du développement historique des entreprises à forte croissance est celui-ci : leur développement est stable à travers le temps, et ce, en dépit du fait que pendant la période visée, l'économie a connu la fluctuation conjoncturelle la plus marquée depuis la Grande Dépression. Selon nous, il s'agit là d'un résultat très évocateur qui peut nourrir le débat sur l'écologie des populations contre l'adaptation stratégique. Les entreprises créent-elles leur propre avenir ou sont-elles soumises aux changements dans l'environnement? Dans nos analyses, les entreprises à forte croissance apparaissent comme un groupe capable d'influer sur son avenir. Il est possible d'argumenter que leur développement agrégé est le résultat d'une proaction, vers un but et vers des occasions d'affaires telles qu'on les décrit dans la documentation sur l'entrepreneuriat. Lorsque l'économie connaît un ralentissement, elles poursuivent leur développement presque comme avant, tandis que lorsqu'il y a reprise, elles n'augmentent pas nécessairement beaucoup plus ( $c f$. l'entreprise individuelle) leur rythme de croissance. Donc, peu importe la situation, elles trouvent des moyens pour atteindre leurs buts. Pendant les années plus difficiles, une croissance à l'interne peut être plus ardue à réaliser; par conséquent, on opte pour la croissance par acquisition. Cela indique que certains dirigeants d'entreprises sont capables de contrôler le destin de leurs entreprises.

Nos analyses de la croissance interne versus la croissance par acquisition devraient mettre en exergue, au profit des chercheurs, l'importance cruciale des définitions et des mesures. Ces résultats indiquent en outre que la croissance prend diverses formes qui, à leur tour, ont divers impacts sur l'économie générale. L'intérêt politique pour la croissance rapide et forte est souvent relié à la création d'emplois. Nos résultats révèlent que plusieurs entreprises à forte croissance ne créent pas de nouveaux emplois, mais prennent de l'expansion grâce aux acquisitions. Cette distinction entre croissance interne et croissance par acquisition nous incite à remettre en question l'intérêt politique de se concentrer sur la croissance d'entreprises individuelles plutôt que sur celle des branches industrielles. Suivant nos résultats, on devrait plutôt mettre l'accent sur le renouvellement, soit l'entrée d'un grand nombre de nouvelles entreprises dans des branches jeunes et en expansion.

\section{Les limites de cette étude}

Il existe deux problèmes que nous n'avons pas pu résoudre à notre satisfaction. Premièrement, beaucoup d'entreprises peuvent choisir de poursuivre une croissance en créant un groupe d'entreprises. Dans notre échantillon complet, 1672 (14,2\%)

Revue internationale P.M.E., vol. 14, $\mathrm{n}^{\text {os }} 3-4,2001$

(C) 2002 - Presses de l'Université du Québec

Édifice Le Delta I, 2875, boul. Laurier, bureau 450, Sainte-Foy, Québec G1V 2M2 • Tél. : (418) 657-4399 - www.puq.uquebec.ca

Tiré de : Revue internationale P.M.E., vol. 14, nos 3-4, sous la direction de Pierre-André Julien. 
entreprises étaient dirigeantes d'un groupe en 1996. Au total, 1372 entreprises $(11,7 \%)$ s'affilièrent à un groupe pendant les dix années d'observation ; il est clair que cela peut cacher plusieurs activités à forte croissance. Deuxièmement, plusieurs entreprises peuvent afficher une croissance sur le plan du chiffre d'affaires, mais pas sur celui du nombre d'employés, en augmentant le recours à la sous-traitance. Des données sur la croissance du chiffre d'affaires ont été ajoutées à notre base de données. Toutefois, les analyses de ces données ne changent pas nos principales conclusions au regard de l'emploi dans l'entreprise (Davidsson et Delmar, 2000 ; Delmar, Davidsson et Gartner, à paraître).

On peut aussi penser que la période d'observation ait pu avoir une influence sur nos résultats. Au cours de cette période, on a connu une très forte récession à laquelle les grandes entreprises ont réagi en diminuant leurs effectifs et, en parallèle, elles ont eu tendance à recourir à l'externalisation plutôt qu'à la croissance interne. Nous reconnaissons que cela peut faire apparaître les EFC de grande taille et bien établies comme «en croissance par acquisition » plutôt qu' " en croissance interne », ce qui pourrait être le cas dans une autre période. Toutefois, l'impression que la croissance interne se transforme en croissance par acquisition lorsque l'entreprise vieillit et prend de l'expansion est théoriquement justifiée sur plusieurs aspects, d'où notre conviction que ces résultats ne sont pas dus à des particularités de la période étudiée.

AnNexe I

Développement annuel des groupes

\begin{tabular}{lcccccccccccc}
\hline $\begin{array}{l}\text { Compte } \\
\text { (\% de 1996) }\end{array}$ & $\mathbf{1 9 8 7}$ & $\mathbf{1 9 8 8}$ & $\mathbf{1 9 8 9}$ & $\mathbf{1 9 9 0}$ & $\mathbf{1 9 9 1}$ & $\mathbf{1 9 9 2}$ & $\mathbf{1 9 9 3}$ & $\mathbf{1 9 9 4}$ & $\mathbf{1 9 9 5}$ & $\mathbf{1 9 9 6}$ \\
\hline Groupe témoin & 8095 & 8501 & 8870 & 9195 & 9487 & 9800 & 10110 & 10310 & 10493 & 10595 \\
& $(76,4)$ & $(80,2)$ & $(83,7)$ & $(86,8)$ & $(89,5)$ & $(92,5)$ & $(95,4)$ & $(97,3)$ & $(99,0)$ & $(100)$ \\
EFC & 467 & 509 & 547 & 591 & 658 & 760 & 942 & 1103 & 1153 & 1153 \\
& $(40,5)$ & $(44,1)$ & $(47,4)$ & $(51,3)$ & $(57,1)$ & $(65,9)$ & $(81,7)$ & $(95,7)$ & $(100)$ & $(100)$ \\
\hline
\end{tabular}

\section{Bibliographie}

Aiginger, K. et G. Tichy (1991), «Small firms and the merger mania », Small Business Economics, vol. 3, p. 83-102.

BALDWIN, J. et G. PICOT (1995), «Employment generation by small producers in the Canadian manufacturing sector», Small Business Economics, vol. 7, p. 317-331.

BIRCH, D. (1979), The Job Generation Process, Rapport final de Economic Development Administration, Cambridge, MA, MIT Program on Neighborhood and Regional Change. 
BIRCH, D.L. et J. Medoff (1994), «Gazelles », dans L.C. Solmon et A.R. Levenson (dir.), Labor Markets, Employment Policy and Job Creation, Boulder, Westview Press.

BLIXT, L. (1997), Tillväxtföretag i Sverige (Growth companies in Sweden), Cahier de recherche, Stockholm, NUTEK.

DAVIDSSON, P. (1995), «SMEs and job creation in Sweden », manuscrit préparé pour l'OCDE secrétariat / Working Party on SMEs / the Ad Hoc Group on SME statistics.

DAVIDSSON, P. (1997), « High-growth SMEs : feasibility analysis for the Swedish study », Miméo préparé pour le Comité sur les PME de l'OCDE, Paris, OCDE / Jönköping, Jönköping International Business School.

DAVIDSSON, P. et F. DELMAR (2000), «På jakt efter de nya arbetstillfällena : tillväxtföretagens roll (Hunting for new employment: the role of high-growth firms) », Ekonomisk Debatt, vol. 28, p. 267-276.

DAVIDSSON P. et M. HENREKSSON, «Institutional determinants of the prevalence of startups and high-growth firms : evidence from Sweden», Small Business Economics (à paraître).

DAVIDSSON, P., L. LINDMARK et C. OlOFSSON (1993), Business Dynamics in the Swedish Economy: A Regional Perspective, Rapport soumis à la Commission économique de la CEE.

DAVIDSSON, P., L. LindMARK et C. OlOFSSON (1994), Dynamiken i svenskt näringsliv (Dynamisme des entreprises en Suède), Lund, Studentlitteratur.

DAVIDSSON, P., L. LiNDMARK et C. OlOFSSON (1995), «The trend towards smaller scale during the 1980's : empirical evidence from Sweden », Communication présentée au ICSB's 40th World Conference, Sydney, juin.

DAVIDSSON, P., L. LINDMARK et C. OLOFSSON (1996), Näringslivsdynamik under 90-talet (Dynamisme des entreprises dans les années 1990), Stockholm, NUTEK.

DAVIDSSON, P., L. LINDMARK et C. OLOFSSON (1997a), « The extent of overestimation in small firm job creation : an empirical examination of the regression bias », Small Business Economics (à paraître).

DAVIDSSON, P., L. LiNDMARK et C. OLOFSSON (1997b), «SMEs and job creation during a recession and recovery », dans Z. Acs, B. Carlsson et C. Karlsson (dir.), SMEs, Entrepreneurship and the Macro Economy, Cambridge University Press (à paraître).

DAVIS, S.J., J. HALtiWANGER et S. SCHUH (1993), Small Business and Job Creation: Dissecting the Myth and Reassessing the Facts, Cahier de recherche $\mathrm{n}^{\circ} 4492$, Cambridge, MA, National Bureau of Economic Research.

DAVIS, S.J., J. HALTIWANGER et S. SCHUH (1996), Job Creation and Destruction, Boston, MA, The MIT Press.

DELMAR, F. (1997), «Measuring growth : methodological considerations and empirical results », dans R. Donckels et A. Miettinen (dir.), Entrepreneurship and SME Research: On Its Way to the Next Millennium, Aldershot, VA, Avebury, p. 190-216.

Delmar, F., P. DAVIDSSON et W. GARTNER (à paraître), «Arriving at the high-growth firm », Journal of Business Venturing.

Revue internationale P.M.E., vol. 14, $\mathrm{n}^{\text {os }} 3-4,2001$

(C) 2002 - Presses de l'Université du Québec

Édifice Le Delta I, 2875, boul. Laurier, bureau 450, Sainte-Foy, Québec G1V 2M2 • Tél. : (418) 657-4399 - www.puq.uquebec.ca

Tiré de : Revue internationale P.M.E., vol. 14, nos 3-4, sous la direction de Pierre-André Julien. 
Dunne, P. et A. Hughes (1996), «Age, size, growth and survival : UK companies in the 1980s », Journal of Industrial Economics, vol. 42, n 2, p. 115-140.

ENSR (1994), The European Observatory for SMEs, Second Annual Report, Zoetermeer, EIM Small Business Research and Consultancy / European Network for SME Research.

Evans, D.S. (1987), «Test of alternative theories of firm growth », Journal of Political Economy, vol. 95, p. 657-674.

Fumagelli, A. et G. MusSATi (1993), «Italian industrial dynamics from the 1970s to the 1980s : Some reflections on the entrepreneurial activity », Entrepreneurship and Regional Development, vol. 5, p. 25-37.

GUNDRY, L.K. et H.P. WELSCH (2001), «The ambitious entrepreneur: high-growth strategies of women-owned enterprises », Journal of Business Venturing, vol. 16, p. $453-470$.

JOHANSSON, D. (1997), The Number and the Size Distribution of Firms in Sweden and Other European Countries, Thèse de doctorat non publiée, Stockholm School of Economics, Stockholm.

Johnson, J., J. BAldwin et C. Hinchley (1997), Successful Entrants : Creating the Capacity for Survival and Growth, No 61-524-XPE, Statistique Canada.

KIRCHHOFF, B. et B. PHILliPS (1988), « The effect of firm formation and growth on job creation in the United States », Journal of Business Venturing, vol. 3, p. 261-272.

LOVEMAN, G. et W. SENGENBERGER (1991), «The re-emergence of small scale production : an international comparison », Small Business Economics, vol. 3, p. 1-37.

OCDE (1987), «The process of job creation and job destruction », Employment Outlook, p. $97-220$.

PHILLIPS, B.D. et B.A. KIRCHHOFF (1989), «Formation, growth and survival, small firm dynamics in the U.S. economy », Small Business Economics, vol. 1, p. 65-74.

PICOT, G. et R. DUPUY (1998), «Job creation by company size : the magnitude, concentration and persistence of job gains and losses in Canada », Small Business Economics, vol. 10, p. 117-139.

SeXton, C. et F. SEAle (1997), Leading Practices of Fast Growth Entrepreneurs, Kansas City, National Center for Entrepreneurship Research.

SpILling, O. (1995), «Do small firms create jobs? », Communication présentée au 6th ENDEC World Conference on Entrepreneurship, Shanghai, décembre.

STATISTICS SWEDEN (SCB) (1995), Nyföretagandet $i$ Sverige 1993 och 1994 (Nouvelles firmes en Suède 1993 et 1994), Statistiska Meddelander F 15 SM 9501, Örebro, SCB, Publishing Service.

STATISTICS SWEDEN (SCB) (1997), Nyföretagandet i Sverige 1995 och 1996 (Nouvelles firmes en Suède 1995 et 1996), Statistiska Meddelander F 15 SM 9701, Örebro, SCB, Publishing Service.

STOREY, D. (1994), Understanding the Small Business Sector, Londres, Routledge. 
STOREY, D. (1995), « Symposium on Harrison's “Lean and mean" : a job generation perspective », Small Business Economics, vol. 7, n 5, p. 5-8.

STOREY, C. (1996), The Ten Percenters - Fast Growing SMEs in Great Britain, Londres, Deloitte \& Touche.

STOREY, D. et S. JOHNSON (1987), Small and Medium-Sized Enterprises and Employment Creation in the EEC Countries: Summary Report, Commission de la CEE, programme de recherche et d'actions sur le développement du marché du travail, étude $n^{\circ} 85 / 407$.

VAESSEN, P. et D. KeEBle (1995), «Growth-oriented SMEs in unfavourable regional environments », Regional Studies, vol. 29, nº 6, p. 489-505.

VYAKARNAM, S., R. JACOBS et J. HANDELBERG (1997), «Formation and development of entrepreneurial teams in rapid growth businesses ", Présentation à la Babson Entrepreneurship Research Conference, Wellesley, MA, mai.

WAGNER, J. (1992), «Firm size, firm growth and persistence of chance. Testing Gibrats's law with establishment data form lower Saxony, 1978-1989», Small Business Economics, vol. 4, p. 125-131.

Revue internationale P.M.E., vol. 14, $\mathrm{n}^{\text {os }} 3-4,2001$ 